\title{
Modernization process and traditional national sports inheritance research based on fuzzy clustering model
}

\author{
Jian Zhang \\ Department of Physical Education, Dalian Polytechnic University, Dalian 116034, China
}

\begin{abstract}
In modernization process, it should put equal emphasis on economic globalization and cultural diversity. But in real life, world economy is like a big net covers global. Chinese traditional culture is the lone individual which suffers impact from western culture. China has been gradually moved toward world sports power, meanwhile Chinese traditional sports events development is not going well. The paper selects martial arts, wrestling, Chinese chess, archery, and shooting five kinds of traditional sports events as research objects. With data statistics in 2010, 2012 and 2013 as evidence, make use of fuzzy clustering model to cluster these five kinds of traditional sports events. The paper clusters them into one kind, overall analyzes it and gets Chinese traditional sports events development is still in slowly rising period. Finally, cluster them into three kinds, which are respectively martial arts, shooting, Chinese chess 、wrestling and archery. By analyzing these three kinds, it gets conclusions that martial arts development is in good status and constantly rising; shooting development is in general status; Chinese chess, archery and wrestling development status remains so bad and they are constantly declining. Based on this, Chinese traditional sports adjustment its orientation will have better prospects.
\end{abstract}

\section{Introduction}

In the context of economic globalization, culture diversity has been mentioned by people for many times. However, Chinese traditional national sports events suffer western competitive sports impact. People in Digital information age seem to loss interest in traditional culture. Since Beijing successfully organizing the 29th Olympic Games in 2008, national fitness awareness has been gradually strengthened, China has gradually moved toward world sports power. At this time, China needs to spread traditional national sports events to places all around the world to promote Chinese soft power, meanwhile; it also makes proper contributions to world culture diversity future [1-3].

In 2013, Wang Hong-Fang and others in the article "Chinese traditional national sports inheritance system construction research under sports power construction", they applied multiple analysis methods, analyzed traditional national sports inheritance necessity from the aspect of constructing sports power. In 2012, Feng Sheng-Gang in the article "Chinese modernization process minorities' traditional sports effective inheritance path empirical research", by consulting documents and other ways, he stated multiple strategies to propel to minorities traditional sports inheritance. In 2012, Liu Jian in the article "Yunnan province minorities' traditional intangible cultural heritage protection and inheritance", took Yunnan province as an example, from the perspectives of historical stage, traditional industrial culture, classified minorities' traditional sports. In 2012, Xiang Kui in the article "Modernization process Chinese traditional national sports development trend and measurement in school sports", took Xiangxi autonomous prefecture traditional national sports development status as an example to make analysis, he found that traditional sports events suffered western culture fiercely impact, meanwhile provided measurement strategies. In 2011, Tang Li-Xu in "Chinese traditional national sports events hierarchy evaluation system and development strategic research", from the perspective of regional difference, utilized theory of hierarchies, strategic theory and other multiple theories to analyze Chinese traditional national sports events structures and showed Chinese traditional national sports events future development status [2-5].

Chinese traditional sports events are many and varied that include go, chess, Cuju, wrestling, polo, martial arts and others. Integrate above analysis, in modernization process and traditional national sports inheritance aspect research, most are analyzing from the aspect of statement. The paper will carry out data analysis on traditional sports representative events in 2010, 2012, 2013. Apply fuzzy clustering model into traditional sports events development, and make classification on five classical traditional sports events, using their development status to represent traditional national sports events development status. Adopt data and diagrams ways, intuitional and objective show modernization process and traditional national sports inheritance aspects problems.

${ }^{a}$ Corresponding author: JianZhang@163.com 


\section{Model establishments}

China is one of world four great ancient civilizations; it has above 5000 years long history and splendid culture. Every traditional sports event represents Chinese laboring people's wisdom. The model selects traditional sports events five kinds of sports events, martial arts, Chinese chess, archery, shooting and wrestling. It consults data in 2010, 2011 and 2012 from Chinese statistical yearbook, makes analysis on the basis of these data; Figure 1 is traditional sports events' wrestling and archery.
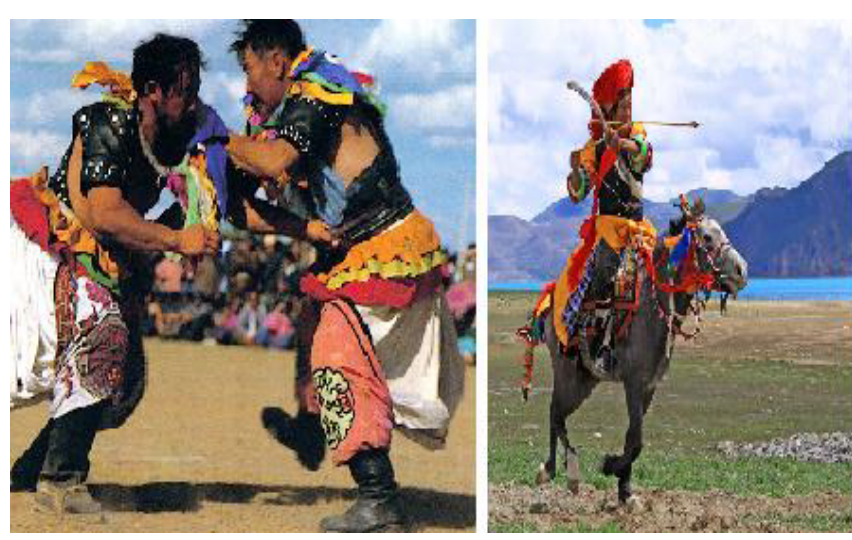

Figure 1. The schematic of traditional sports.

\subsection{Data processing.}

Data comes from Chinese statistical yearbook culture and sports parts technical and event grade development sorted number of athletes. The model establishment selects martial arts, Chinese chess, archery, shooting, Chinese wrestling five kinds of classical traditional events as research objects. Each kind of traditional sports events development number of people is as Table 1 show.

Table 1. Five kinds of traditional sports events development number of people data table.

\begin{tabular}{|c|c|c|c|}
\hline Event & Year 2010 & Year 2011 & Year 2012 \\
\hline Martial arts & 2518 & 2962 & 3533 \\
\hline Chinese chess & 341 & 321 & 248 \\
\hline Archery & 283 & 187 & 219 \\
\hline Shooting & 1018 & 1175 & 1207 \\
\hline Chinese wrestling & 504 & 546 & 556 \\
\hline
\end{tabular}

\subsection{Fuzzy clustering analysis}

In daily life, we tend to need classifying multiple indicators according to certain criterions. But in most cases, classification limits are not very obvious, therefore fuzzy clustering analysis [6] application in practice is very widely. Fuzzy clustering analysis generally has following steps:

Set classified object is $U=\left\{u_{1}, u_{2}, \cdots, u_{n}\right\}$, every object has $m$ pieces of indicators to show its character:

$$
x_{i}=\left\{x_{i 1}, x_{i 2}, \cdots, x_{i m}\right\}, i=1,2, \cdots, n
$$

Therefore it appears matrix

$$
X=\left(x_{i j}\right)_{n \times m}
$$

In real application, different data correspond to different dimensions, to easier comparing data, it needs to normalize data; in the following it introduces some common normalization methods.

$X$ is divided into 4 kinds: $\left\{x_{1}\right\},\left\{x_{2}, x_{3}\right\},\left\{x_{4}\right\},\left\{x_{5}\right\}$;

When it takes $\lambda=0.58$, method is as above. Since space is limited, no more statement. $X$ is divided into 3 kinds: $\left\{x_{1}\right\},\left\{x_{2}, x_{3}, x_{5}\right\},\left\{x_{4}\right\}$

When it takes $\lambda=0.47, X$ is divided into 2 kinds: $\left\{x_{1}\right\},\left\{x_{2}, x_{3}, x_{5}, x_{4}\right\}$

When it takes $\lambda=0.38, X$ is divided into 1 kind: $\left\{x_{1}, x_{2}, x_{3}, x_{5}, x_{4}\right\}$

For above process, use partial dynamical clustering graph to express as Figure 2.

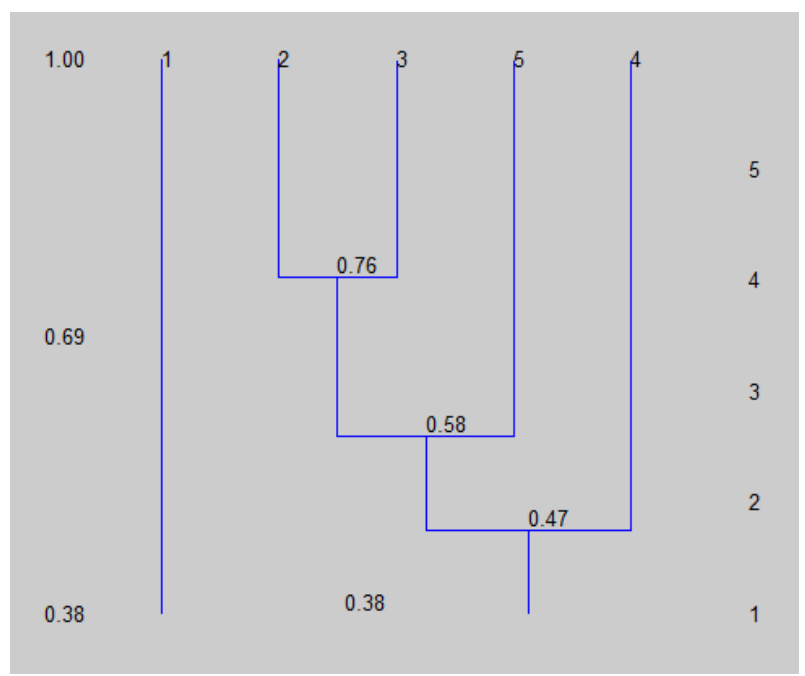

Figure 2. The figure of part of the dynamic clustering.

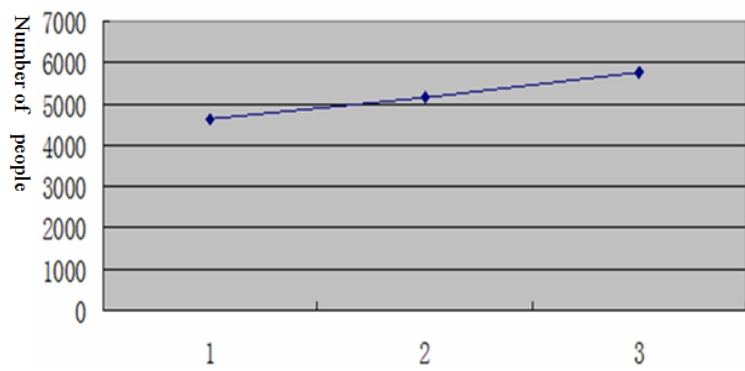

Figure 3. The diagram of overall development.

To consider overall effects, cluster them into one kind to analyze. Figure 3 is their overall development status graph. By Figure 3, it is clear that Chinese traditional sports event overall development status is in slowly rising stage.

By consulting relative documents, it is clear that fuzzy clustering analysis $2 \sim 3$ kinds results are the best, so it selects clustering result when $\lambda=0.1$. 


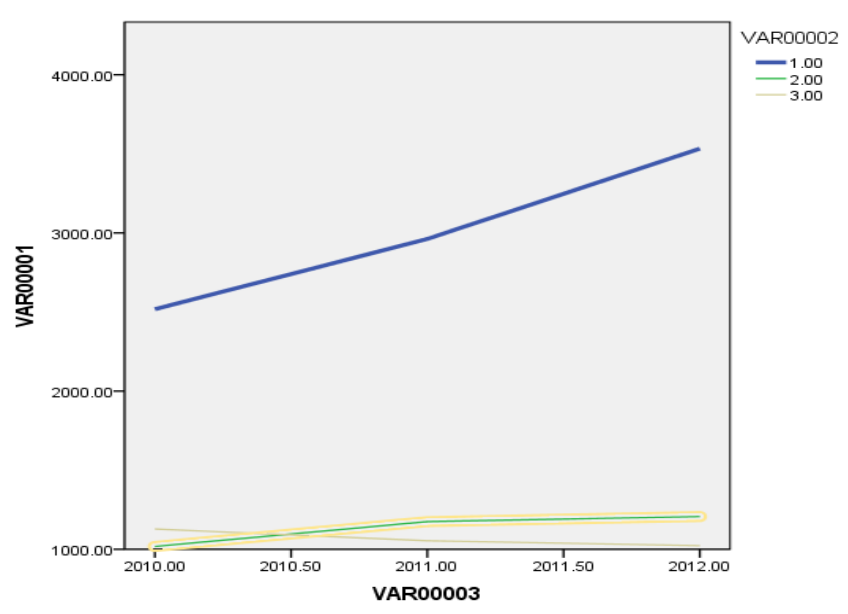

Figure 4. The development of traditional sports.

Figure 4 is after classification traditional sports events development status graph, represents martial arts, represents shooting, and represents Chinese chess, archery and wrestling. By Figure 4, it is clear that martial arts development is good and constantly rising; shooting development is general; Chinese chess, archery and wrestling development remains so bad, and they are constantly declining. Therefore, in modernization development process [7], it should focus on Chinese chess, archery and wrestling and other sports events development, meanwhile it should maintain martial arts good development trend, and strive for promoting shooting's development level.

\section{Conclusion}

Conclusions got by research is that Chinese traditional sports events overall development is good, from which, martial arts development is excellent, but Chinese chess, wrestling and archery development are not going well. Chinese chess recognition degree in international is not enough that causes Chinese chess is difficult to spread to foreign countries. Due to wrestling and archery has certain lethality, they are not advocated by people in daily life. For the above analysis, relative people should consider Chinese chess spreading abroad problems as well as wrestling and archery reform problems.

\section{References}

1. G. Wang, Journal of Capital College of Physical Education, 20, 1-4(2008).

2. J. Zhao, Sports Sciences Researches, 15, 14-17(2011).

3. X. W. Ye, Bulletin of Sport Science \& Technology, 19, 112-113, 125(2011).

4. R. Z. Li and C. G. Yu, Sports Culture Guide, 4, 84-86(2007).

5. Y. G. Hu, Journal of Shenyang Sport University, 28, 110-113, 128(2009).

6. X. Chen, Z. Jin and G. Lin et al, Journal of Coastal Research, 73, 711-719(2015).

7. Z. L. Liu, Advances in Information Sciences and Service Sciences, 4, 210-218(2012). 